\title{
A priori testing of subgrid models for chemically reacting non-premixed turbulent shear flows
}

\author{
By JAVIER JIMÉNEZ $Z^{1,2}$, AMABLE LIÑ Á N ${ }^{2,1}$, \\ MICHAEL M. ROGER S ${ }^{3}$ AND \\ FRA NCISCO J. H I GUERA A $^{2,1}$ \\ ${ }^{1}$ Centre for Turbulent Research, Stanford University, Stanford, CA 94305, USA \\ ${ }^{2}$ School of Aeronautics, Universidad Politécnica, 28040 Madrid, Spain \\ ${ }^{3}$ NASA Ames Research Centre, Moffett Field, CA 94035, USA
}

(Received 3 December 1996 and in revised form 5 April 1997)

The $\beta$-assumed-p.d.f. approximation of Cook \& Riley (1994) is tested as a subgrid model for the LES computation of non-premixed turbulent reacting flows, in the limit of infinitely fast chemistry, for two plane constant-density turbulent mixing layers with different degrees of intermittency. Excellent results are obtained in the computation of plane-averaged properties, such as product mass fractions and relatively high powers of the temperature, and even of the p.d.f. of the conserved scalar itself. In all these cases the errors are small enough to be useful in practical applications. The analysis is extended to slightly out-of-equilibrium problems, such as the generation of radicals, and formulated in terms of the p.d.f. of the gradient of the mixture fraction. It is shown that the form of the conditional gradient distribution is universal in a wide range of cases, whose limits are established. Within those limits, engineering approximations to the radical concentration are also possible. It is argued that the experiments in this paper are already in the limit of high Reynolds numbers.

\section{Introduction}

The computation of turbulent reacting flows is an open challenge even after having been the focus of intensive work for several decades. The subject of this paper, non-premixed flames with fast chemistry, was one of the first to be tackled and it is somewhat simpler than others, but it still represents a large number of cases of theoretical and practical importance. Recent reviews can be found in Bilger (1989), Libby \& Williams (1994).

Our analysis is subject to several simplifications. The diffusion coefficients of all the species and of heat are assumed to be identical and, although not explicitly needed for most of the theoretical arguments, all of our numerical experiments are done at Schmidt number $S c=0.7$. Our flows are incompressible, and we assume that any heat released by the reaction is weak enough for the fluid density to be unchanged, $\rho=1$. The role of the chemistry is thus passive with respect to the flow, but it is modified by it.

In the first part of the paper we assume an irreversible binary reaction

$$
v_{A} \mathrm{~A}+v_{B} \mathrm{~B} \rightarrow v_{P} \mathrm{P}
$$


in a shear flow between two streams, each of which initially contains either A or B reactant.

Under those circumstances the mass fractions $Y_{i}$ of the different species can be linearly combined to form a set of conserved scalars which are transported by the flow with the common diffusion coefficient $\kappa$. If in addition the Damköhler number, which measures the ratio of the characteristic diffusion and chemical times, is large enough, the reaction occurs in a thin flame that can be treated as a surface, and the problem reduces to the mixing of a single conserved scalar

$$
\xi=\frac{1-y_{A}+s y_{B}}{1+s}
$$

called the mixture fraction, which takes values $\xi=0$ and $\xi=1$ at the free streams (see Williams 1985). Here $y_{i}=Y_{i} / Y_{i 0}$, where $Y_{i 0}$ is the mass fraction of the $i$ th species at the appropriate free stream, and

$$
s=v_{A} W_{A} Y_{B 0} / v_{B} W_{B} Y_{A 0},
$$

where $v_{i}$ and $W_{i}$ are stoichiometric coefficients and molecular weights. In this BurkeSchuman limit the flame is located at the stoichiometric mixture fraction

$$
\xi_{s}=(1+s)^{-1} \text {, }
$$

and most quantities of interest can be computed as algebraic functions of $\xi$, which are continuous but have discontinuous derivatives at $\xi_{s}$. Thus the mass fraction of the product is proportional to the triangular function

$$
Y_{P}(\xi)=\left\{\begin{array}{l}
\xi / \xi_{s} \text { if } \xi \leqslant \xi_{s}, \\
(1-\xi) /\left(1-\xi_{s}\right) \text { otherwise. }
\end{array}\right.
$$

In modelling turbulent flows one usually estimates averaged or locally filtered values of $\xi$, and would like to have similarly filtered values of mass fractions or other quantities, but is prevented from doing so by the nonlinear nature of (1.5).

It was soon realized that what is needed is an approximation to the probability density function (p.d.f.) of $\xi$, and that the mean value of any quantity which can be expressed as a function of $\xi$ is (Lin \& O'Brien 1974; Bilger 1976)

$$
\langle f\rangle=\int f(\xi) p(\xi) \mathrm{d} \xi,
$$

where $p(\xi)$ is the p.d.f. Numerous experimental (LaRue \& Libby 1974; Anselmet \& Antonia 1978; Breidenthal 1981; Mungal \& Dimotakis 1984; Koochesfahani \& Dimotakis 1986), theoretical or numerical (Eswaran \& Pope 1988; Pumir 1994; Holzer \& Siggia 1994), and modelling (Kollman \& Janicka 1982; Broadwell \& Breidenthal 1982) efforts have been undertaken to understand the properties of the p.d.f. of passively mixed scalars.

Of particular interest in this paper is the $\beta$-p.d.f. model of Cook \& Riley (1994), in which the form of the scalar p.d.f. is modelled as a function of its mean value and standard deviation and, specially, its use as a sub-grid model for large-eddy simulations (LES). Large-eddy simulation has proved to be a powerful technique for the computation of complex flows and good results have been obtained in the computation of filtered scalar mean values (Lesieur \& Rogallo 1989; Moin et al. 1991). We will show below that the subgrid fluctuation intensity can also be estimated with good accuracy. In this paper we will assume that exact filtered mean scalar 
values can be computed by some LES scheme, but we will obtain them by filtering direct numerical simulation fields. The $\beta$-p.d.f. model has been tested in this way for isotropic turbulent flow at relatively low Reynolds numbers in Cook \& Riley (1994) and Réveillon \& Vervisch (1996). We will test it here in the more realistic case of a mixing layer at medium Reynolds numbers (Rogers \& Moser 1994).

At issue is the question of large-scale turbulent intermittency, which is the presence of essentially laminar pockets in an otherwise turbulent flow, and whether the same subgrid mixing model can be used in homogeneous turbulence and in the presumed interface between turbulent and laminar regions. A lot of effort has gone into modelling such intermittency effects (Libby 1975; Dopazo 1977; Kollman 1984; Pope 1985; Pope \& Correa 1988) but, if they are really large-scale phenomena, LES should be able to resolve them without resorting to modelling. The main difference between homogeneous flows and the mixing layer is that, while large-scale intermittency is rare in the former, it is prevalent in the latter.

The simulation experiments are described in the next section. The results of applying the $\beta$-p.d.f. model to the prediction of different quantities in infinitely fast chemistry are presented in $\$ 3$. We then extend the model to finite-rate chemistry in the flamelet limit, and introduce some results on the joint p.d.f. of the scalar and the scalar gradients, followed by discussion and conclusions.

\section{Numerical experiments}

The two flow fields used in this report are taken from the simulations in Rogers \& Moser (1994), where they are described in detail. Briefly, they are direct simulations of three-dimensional temporally growing mixing layers, spatially periodic in the streamwise and spanwise directions, with initial conditions which represent turbulent boundary layers. The flow fields chosen are those in figures 18.a and 18.c of that paper, in which the momentum thickness, $\theta$, of the layers have grown by factors of 2.47 and 2.94 respectively from the initial conditions. The streamwise integral scales, after decreasing slightly as the initial boundary layers relax to a shear layer profile, have increased by a factor of about 4 . The energy spectra have a short power-law range with an exponent close to $-5 / 3$, and the layers are growing self-similarly. Both layers appear to be slightly beyond the 'mixing transition' identified in Konrad (1976) and Breidenthal (1981). The ratio between vorticity and momentum thickness is about 4.85 in both cases.

The Reynolds numbers based on the instantaneous momentum thickness are 1980 and 2350, corresponding to longitudinal microscale Reynolds numbers $R e_{\lambda}=127$ and 214 at the central plane of the layer. Note that both flows are intermittent, especially the second one, and that these values of $R e_{\lambda}$ would be be somewhat reduced if they were conditioned only to the turbulent fluid. The effect can be estimated roughly by noting that $R e_{\lambda} \sim u^{\prime 2} / \epsilon^{1 / 2}$, where $\epsilon$ is the turbulent energy dissipation rate, and assuming that, while the turbulent energy is not subject to intermittency effects, the dissipation is concentrated in the turbulent fluid and is proportional to the intermittency coefficient $\gamma$. Defining a conditioned dissipation $\epsilon_{t}=\epsilon / \gamma$, it follows that the conditioned, 'turbulent', Reynolds number would be $R e_{\lambda t}=\gamma^{1 / 2} R e_{\lambda}$. Taking $\gamma$ from figure 1 below, it follows that the reduction in Reynolds number would be negligible in the first case, and of the order of $10 \%$ is the second, leaving both flows among those with the highest $R e_{\lambda}$ in available numerical computations, and in the range of many experimental investigations on mixing.

The computational boxes are, in both cases, $125 \times 31.25$ initial momentum thick- 


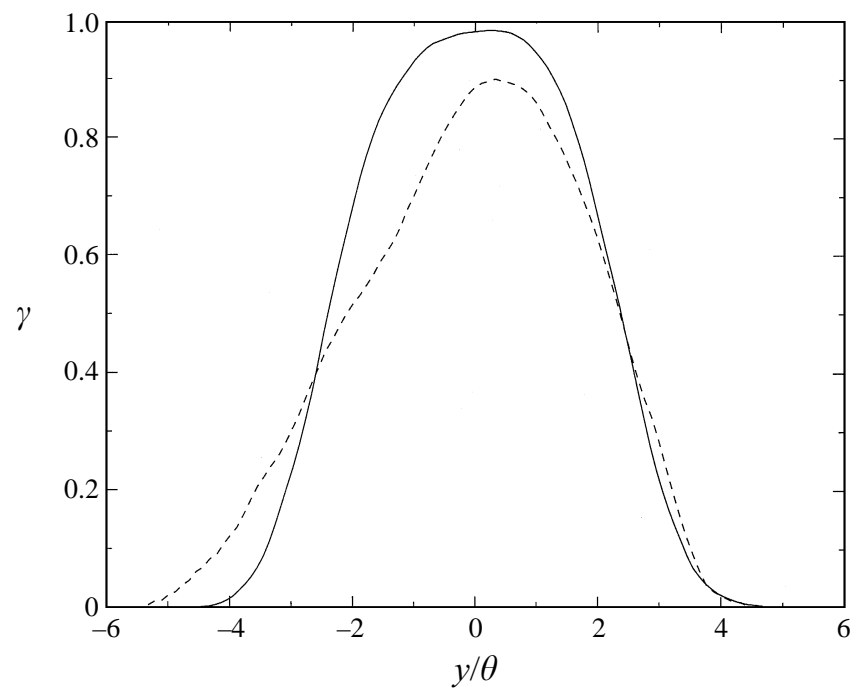

FIGURE 1. Scalar intermittency across the two mixing layers used in the text, defined as the fraction of fluid for which $\xi \in(0.02,0.98)$ : — , unforced case; - - , forced.

ness, and contains five or six large spanwise structures at the times chosen for our experiments. A passive scalar is introduced at the initial condition with a laminar profile of thickness similar to that of the vorticity layer, and a range $\xi \in(0,1)$. Although the original simulations were spectral, using a mesh that was neither uniform nor isotropic, they were spectrally interpolated to physical variables on a uniform isotropic mesh for the purpose of our experiments. This implies some reduction in the resolution at the centre of the layer, which is dictated by the least-resolved direction. Thus while the original computations were carried using $512 \times 180 \times 128$ and $384 \times 96 \times 96$ spectral modes, the interpolated fields contain $512 \times 128 \times 128$ and $384 \times 120 \times 128$ points. The pitch of the interpolated grids is $\Delta x / \theta \approx 0.1$ in both cases, although the original grids are finer by a factor of about 2, especially at the central plane and in the transverse, $y$, direction. All lengths in this report are normalized with the instantaneous momentum thickness of the layers. In terms of the Kolmogorov scale at the centre of the layer, $\theta / \eta=67$ and 72 respectively, and the resolution of our interpolated grid is about $7 \eta$ in both cases.

It was found in Rogers \& Moser (1994) that the structure of the layer depends during the whole simulation on the initial conditions, corresponding to similar longterm effects during the initial development of experimental layers. Different amounts of initial perturbations were introduced in the simulations to mimic this effect. Our two flow fields correspond to two extreme cases in the amount of two-dimensional perturbations applied to the initial conditions. In the first one, which will be referred from now on as the 'unforced' case, the initial conditions were synthesized from two turbulent boundary layers without modification. At the time of our experiment, both the vorticity and the scalar field are fairly disorganized with weak spanwise-coherent structures, and there is very little fresh fluid at the centre of the layer. In contrast, the second ('forced') case was initialized by amplifying the spanwise-coherent modes of the initial boundary layers by a factor of 20 , and the resulting two-dimensional forcing gives rise to clear spanwise rollers with fresh fluid from both streams present across the layer. This is clear in figure 1, which presents the fraction of mixed fluid 
in both cases, arbitrarily defined as $\xi \in(0.02,0.98)$. Not only is the mixed fraction higher in the unforced case, but the presence of just a few large structures in the forced one results in poor statistics, which are not symmetric with respect to the central plane. The statistics for the unforced case are symmetric.

Of the two cases, the forced one is the hardest to model because of the higher intermittency. Most of the results given below are for this case. The corresponding ones for the unforced case are at least as good, and usually better.

We will generally compare mean quantities, denoted by $\langle\cdot\rangle$, which are obtained by averaging over whole $(x, z)$-planes. Occasionally the averages will be extended to slabs of the mixing layer, in which case the limits of the transverse coordinate $y$ will be given explicitly. In our simulations of LES we define our basic filtering operation as a box filter in physical space. Quantities are averaged over a cubical box of contiguous grid points of side $h=n \Delta x$, and assigned to the centre of the box. This operation will be denoted by an overbar. Other filtering kernels have been used by other investigators, and it is not clear which is the best choice to mimic the projection operation implicit in the use of a discrete grid, but our choice seems natural for finite-differences or finite-volumes codes, and has the advantage of providing a simple definition for subgrid statistics.

Equation (1.6) extends trivially to filtered quantities, but the p.d.f. has to be understood as referring only to the interior of the filter box. For a filter of width $h$, filtered quantities can be interpreted as averages with respect to a subgrid p.d.f. $p_{h}$, which is defined by requiring that, for any function of $\xi$,

$$
\bar{f}(\boldsymbol{x})=h^{-3} \int_{h^{3}} f\left[\xi\left(\boldsymbol{x}-\boldsymbol{x}^{\prime}\right)\right] \mathrm{d}^{3} \boldsymbol{x}^{\prime}=\int f\left(\xi_{1}\right) p_{h}\left(\xi_{1} ; \boldsymbol{x}\right) \mathrm{d} \xi_{1} .
$$

In particular, we define in this way the filtered $\bar{\xi}$ and the subgrid variance

$$
\xi_{h}^{\prime 2}=\overline{\xi^{2}}-\bar{\xi}^{2}
$$

In defining the p.d.f. in the rightmost part of (2.1), we have substituted the geometric filtering in the first part of the equation by a probabilistic averaging over a fictitious random variable $\xi_{1}$, which has a uniform value inside the filter box for any given realization. Thus while $\boldsymbol{x}^{\prime}$ in the first integral in (2.1) is a label for the geometric distribution of $f$, the variable $\boldsymbol{x}$ in the second one is a label for the filter box, and $\xi_{1}$ is a variable instead of a field. That substitution is the same that underlies (1.6), and its validity is equivalent to assuming ergodic properties for the subgrid field.

For a given grid element in a given simulation, the $p_{h}$ defined in this way is of course only an estimation of the subgrid p.d.f., and a consistent definition would require a statistical sample of equivalent grid elements. The assumption of which grid elements should be considered equivalent underlies the different subgrid models, even before a particular form is given for the p.d.f. Thus the simpler models in which the subgrid properties are parameterized only with the value of the filtered scalar are based on the assumption that all the grid elements with a given $\bar{\xi}$ have a common subgrid p.d.f., while the more complex ones discussed in $\$ 3.2$ assume that only those elements with the same $\bar{\xi}$ and $\xi_{h}^{\prime}$ are statistically equivalent to each other. A more complete discussion of this point would take us too far from the purpose of this paper. We will drop from now on the explicit distinction between $\xi$ and $\xi_{1}$, although their conceptual difference should be kept in mind when interpreting some of the formulas below.

All the averaged quantities are functions of $y$ and, in addition, the filtered quantities 
are also functions of the homogeneous coordinates, $x$ and $z$, and time. To increase the number of data points available for the statistics, filtered quantities are computed at all grid points, even if they are only strictly independent over a coarser grid of pitch $h$. Plane averages are then computed for these filtered quantities and used to generate filtered profiles, which satisfy

$$
\langle\bar{f}\rangle=h^{-1} \int_{-h / 2}^{h / 2}\langle f\rangle\left(y-y^{\prime}\right) \mathrm{d} y^{\prime}=\overline{\langle f\rangle} .
$$

Note that we can combine (1.6) and (2.3) to generate a 'filtered' p.d.f. for $\xi$,

$$
\bar{p}(\xi, y)=h^{-1} \int_{-h / 2}^{h / 2} p\left(\xi, y-y^{\prime}\right) \mathrm{d} y^{\prime}=\left\langle p_{h}\right\rangle,
$$

where the last equality follows from averaging (2.1) over a plane and using (2.3). Equation (1.6) generalizes to

$$
\langle\bar{f}\rangle=\int f(\xi) \bar{p}(\xi) \mathrm{d} \xi,
$$

and the error of any approximation to the mean profiles depends on our success in approximating $\bar{p}$ from our local models for $p_{h}$. In most of our experiments the filter width is small enough with respect to the width of the layer that we will be able to neglect the difference between laterally filtered and unfiltered p.d.f.s.

\section{Fast chemistry}

\subsection{No subgrid model}

It should be clear from the discussion in the last section that the aim of any approximation should be to reproduce $\bar{p}(\xi)$ as closely as possible. In RANS computations, the only available information is the mean value of the mixture fraction over a plane and perhaps some of its statistical moments. Unless some model is applied for the form of the p.d.f., the implied representation is a delta function $\bar{p}=\delta(\xi-\langle\xi\rangle)$, which is known to be poor.

In LES we have some hope of avoiding such modelling, since the grid elements are small parts of the flow which may be assumed to be fairly well mixed, with small fluctuations with respect to the local filtered value. Large intermittent unmixed regions hopefully span individual grid elements. In this approximation

$$
p_{h}(\xi)=\delta(\xi-\bar{\xi}), \quad \overline{f(\xi)}=f(\bar{\xi}) .
$$

In practice the filtered grid values are treated as points and used to compile the statistics.

The approximation (3.1) is tested directly in figure 2 for the p.d.f.s of the mixture fraction in two planes of the forced mixing layer. Each figure contains the p.d.f.s resulting from several filters, compared to the real one. The widths of the filters are of the order of 30-50 Kolmogorov lengths, comparable to the momentum thickness, and correspond to grids of 10-20 points across the layer (figure 1). Even with these relatively coarse grids the approximation to the p.d.f. is much more realistic than a delta function, and captures the general shape of the real p.d.f. Product mass fraction profiles obtained using these p.d.f.s in (2.5) have errors of the order of $20 \%$.

Nevertheless there are clear differences between the true and the approximate p.d.f.s. A sizeable percentage of the pure fluid that should be included in the delta 

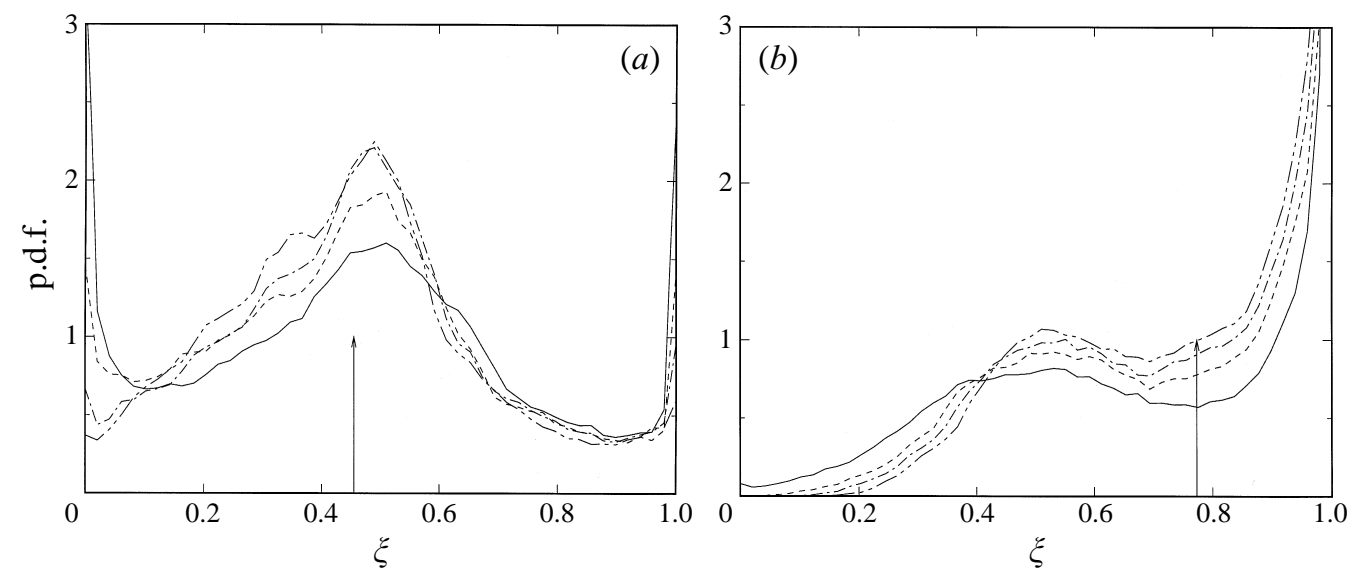

FIGURE 2. Mixture fraction p.d.f.s from LES without subgrid modelling. Forced layer. (a) $y / \theta=0$, (b) $y / \theta=3:-$, no filter;,$--- h / \theta=0.44 ;-\cdot-, 0.66 ;-\cdot--, 0.88$. Vertical arrows mark the mean value of $\xi$ for each plane.

functions at $\xi=0$ and $\xi=1$ has been aliased as mixed fluid in the central peak. This is especially evident deep into the layer (figure $2 a$ ) where the unmixed regions are presumably of small size and are almost completely obliterated by the filter.

\subsection{The $\beta$ subgrid model}

It was noted by Cook \& Riley (1994) that the approximation in the previous section could be improved if the subgrid variance (2.2) were known at each grid point. It would then be possible to make a reasonable guess as to the form of the subgrid p.d.f., $p_{h}\left(\xi ; \bar{\xi}, \xi_{h}^{\prime}\right)$, and to obtain a better estimate of the true p.d.f. in terms of the joint p.d.f. of those two subgrid variables using

$$
\bar{p}(\xi)=\int p\left(\bar{\xi}, \xi_{h}^{\prime}\right) p_{h}\left(\xi ; \bar{\xi}, \xi_{h}^{\prime}\right) \mathrm{d} \bar{\xi} \mathrm{d} \xi_{h}^{\prime},
$$

which is equivalent to the second equation in (2.4) when $p_{h}$ is labelled by $\bar{\xi}$ and $\xi_{h}^{\prime}$ instead of by $\boldsymbol{x}$.

In the particular model proposed in that paper, the subgrid p.d.f. is represented as a $\beta$-distribution, $p_{h} \sim \xi^{a-1}(1-\xi)^{b-1}$, and the two exponents $a$ and $b$ are computed at each point from the values of $\bar{\xi}$ and $\xi_{h}^{\prime}$.

This correction needs the subgrid scalar variance, $\xi_{h}^{\prime}$, which is generally not given by the LES equations, but the same paper suggests that it may be obtained by a similarity argument from the behaviour of the scalar at scales close to the grid filter. Consider in our implementation two filter levels (figure $3 a$ ). The first one is the grid filter of width $h$, which is represented by the dashed squares. The test filter $\bar{\xi}$ is formed by averaging a $2^{3}$ cubic box of contiguous, non-overlapping, grid values. In this implementation

$$
\widehat{\bar{\xi}}=\widehat{\xi}
$$

and we can define the subgrid variance at the test level

$$
\xi_{2 h}^{\prime 2}=\widehat{\xi}^{2}-\widehat{\xi}^{2} .
$$

Neither (3.4) nor (2.2) are known, but they can be combined to give a band-passed 

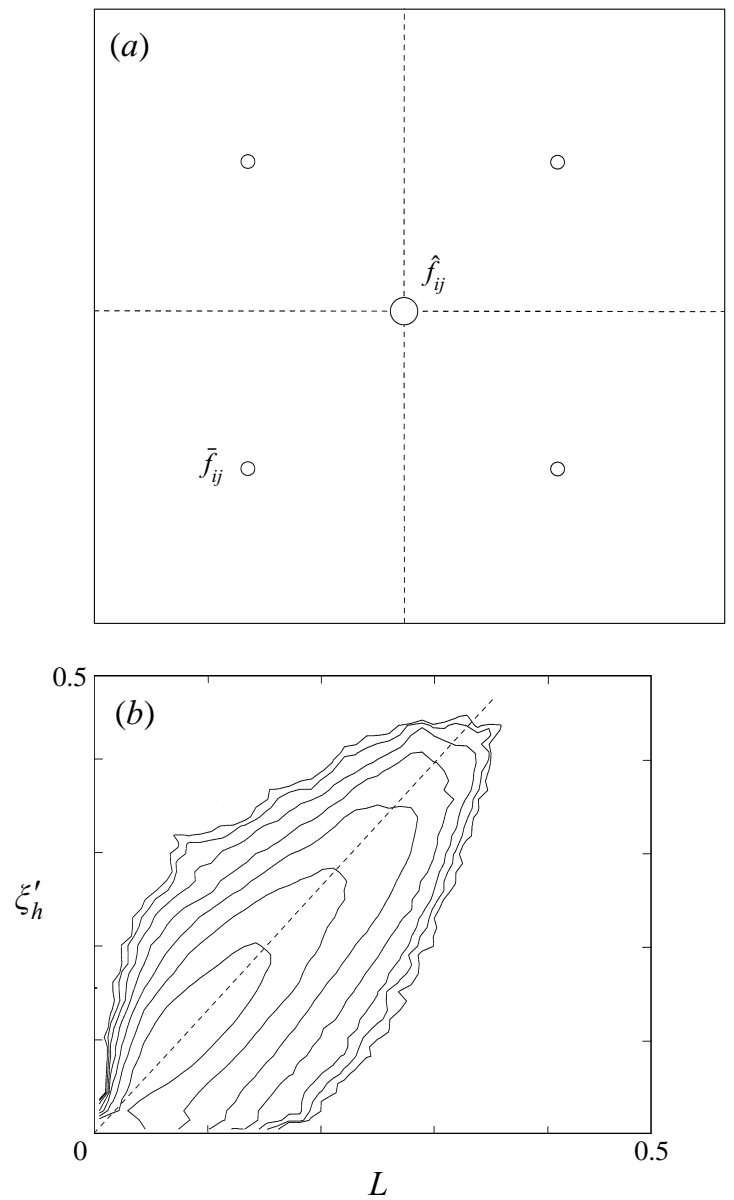

FIGURE 3. (a) Sketch of the two filters for the estimation of the subgrid variance. (b) Joint p.d.f. of the band-passed mixture fraction fluctuation $L$ and its true subgrid value $\xi_{h}^{\prime}$. The dashed line is (3.7) with spectral slope $-5 / 3$. Isolines are logarithmically spaced by half an order of magnitude. Forced layer. $y / \theta=(-2,2)$.

'Leonard' term which, using (3.3), can be written as

$$
L^{2}=\xi_{2 h}^{\prime 2}-\widehat{\xi}_{h}^{\prime 2}={\widehat{\bar{\xi}^{2}}}^{2} \widehat{\bar{\xi}}^{2} .
$$

The right-hand side involves only filtered quantities, and can be computed as the standard deviation of $\bar{\xi}$ within the box defining the test filter. The similarity assumption is that

$$
\xi_{h}^{\prime}=c_{L} L
$$

and is seen to be reasonable in figure $3(b)$, where it is tested for the central part of the forced layer.

The proportionality constant can be estimated by assuming a form for the scalar spectrum, $S(k) \sim k^{-\beta}$. The subgrid variance is obtained by filtering the spectrum through the transfer function of the filter, which has the form $F(k h)$. The result is that $\xi_{h}^{\prime 2} \sim h^{\beta-1}$ and, from (3.6)

$$
c_{L}=\left(2^{\beta-1}-1\right)^{-1 / 2}
$$



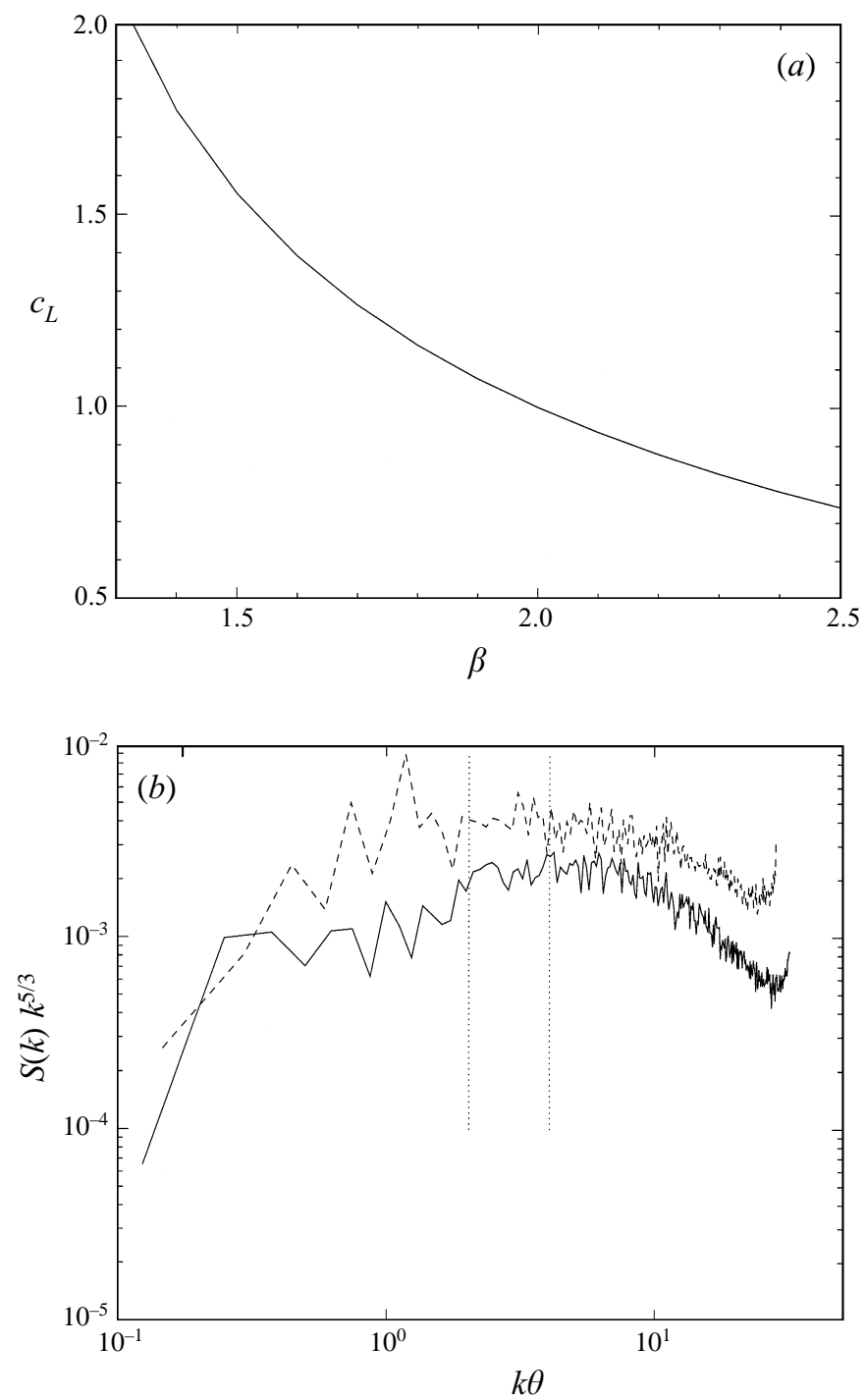

Figure 4. (a) Proportionality constant between band-passed and subgrid fluctuations, versus spectral slope. (b) Compensated scalar longitudinal spectra at the central planes (arbitrary units).

Unforced layer; - - , forced. Vertical doted lines are $k h=\pi$ for the two filters used in figure $3(b)$.

This quantity, which assumes an infinite Reynolds number in that it integrates the spectrum to $k \rightarrow \infty$, is plotted as a function of the spectral slope in figure $4(a)$. For the Kolmogorov slope $\beta=5 / 3$ it has a value $c_{L}=1.305$, which is the one used for the dashed line in figure $3(b)$, and represent the data well. In reality it is known that scalar spectra have slopes which are somewhat shallower than $5 / 3$ for Reynolds numbers in the range of our experiments (see Sreenivasan, 1996 and figure $4 b$ ). This would imply proportionality constants somewhat higher than our value, but this effect is partly compensated by the presence of a Kolmogorov cutoff in the spectrum, which would lead to a lower value of $c_{L}$. Figure $4(b)$ show our scalar spectra, and the position of our filters with respect to them. The fact that both effects compensate at our Reynolds number, and that they should vanish as $R e \rightarrow \infty$, suggests that the asymptotic value 

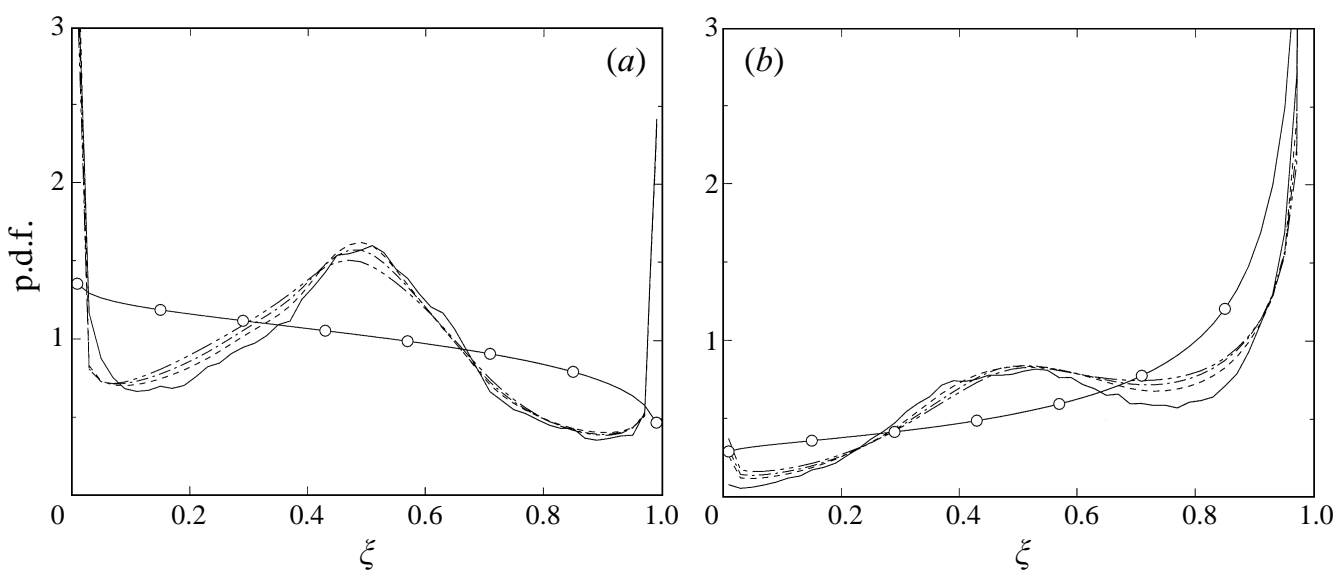

FIGURE 5. Mixture fraction p.d.f.s from LES using the similarity $\beta$ subgrid model. Forced layer. $(a)$ $y / \theta=0$, (b) $y / \theta=3:-$, no filter;,$--- h / \theta=0.55 ;-\cdot-, 0.77 ;-\cdot \cdot-, 0.99 ;-\circ-, \beta$-distributions using the global mean and standard deviation for each plane.

of $c_{L}$ is a reasonable approximation for most of the Reynolds numbers of interest in LES.

In the two previously published tests of the $\beta$-p.d.f. model, the proportionality constant $c_{L}$ was fitted to the data and found to be smaller than ours. J. Réveillon $\&$ L. Vervisch (1996, private communication) found $c_{L}=0.5$ for a filter ratio of 2 , while Cook \& Riley (1994) found $c_{L} \approx 1$ for $\widehat{h} / \bar{h}=1.8$, which would correspond to $\beta \approx 2.15$ according to a calculation similar to (3.7), and to $c_{L} \approx 0.9$ for $\widehat{h} / \bar{h}=2$. Both simulations, however, were carried at Reynolds numbers substantially lower than ours. Réveillon \& Vervisch worked at $R e_{\lambda}=17$, for which there is no inertial range and no self-similar spectrum, and where turbulence is barely developed. Cook \& Riley do not give their Reynolds number, but their filters are only six times larger than the Batchelor scale, which would be near the right-most points in the spectra in figure 4, and within the dissipative range. Neither experiment can therefore be expected to agree with an inertial-range prediction. It is probably a general rule that, if LES models are to behave independently of the type of flow, they should only be used in well-developed turbulence, with filters in the inertial range. In calculations like the present one, involving advected scalars, the scalar spectrum should also be well-developed. The two conditions are probably equivalent for mixing flows with $S c \approx 1$, where turbulence and mixing develop simultaneously, but would result in different constraints if, for example, sharp scalar gradients were injected in previously established turbulence. The present paper implicitly assumes that there is a shared range of scales in which the velocity and the scalar fluctuations follow an inertial behaviour, and that the LES filters are chosen in that range.

The results of applying the $\beta$-correction to the p.d.f. in the previous section are shown in figure 5, where it is seen that the error has decreased considerably with respect to figure 2 and, in particular, that it is now relatively insensitive to the filter width. In computing this and the following figures, the values of $\bar{\xi}$ are obtained by filtering the direct numerical simulation fields at two different resolutions. The subgrid variances are estimated using (3.6), and the p.d.f. is obtained by substituting the resulting $\beta$-distributions for $p_{h}$ in (3.2). Note that the good behaviour of the model is not only at the level of integral quantities, but at the detailed level of the 
p.d.f., implying that it should give good results for the average of any function of $\xi$ and not only for the product mass fraction (1.5). This includes the approximation of the p.d.f. at a particular value of $\xi$, which is useful, for example, in evaluating source terms located at the flame. The figure includes the $\beta$-distributions corresponding to the global averages and (true) standard deviations at each plane, as would be used in Reynolds-averaged numerical simulation (RANS).

Although they are not included in the figure, there is no appreciable difference between the LES results obtained using the value of $\xi_{h}^{\prime}$ evaluated directly from the DNS data, and those obtained using the similarity assumption (3.6).

It is curious that, when the RANS p.d.f.s are used to compute the mean value of the product mass fraction (1.5), the result is within a few percent of the actual one, but it is clear from figure 5 that this is due to compensating errors, and that it cannot be extrapolated to other quantities.

\subsection{Mean profiles}

The results of using the approximate p.d.f.s of the previous section to compute mean profiles of various quantities are presented in figure 6 , in which the degree of difficulty increases from top to bottom and from the left to the right. Plots $(a, c, e)$ are computed for a stoichiometric mass fraction $\xi_{s}=1 / 2$, for which the flame is roughly in the middle of the mixing layer. There the fluid is relatively well mixed, and the results should be comparable to those obtained in homogeneous turbulence. Plots $(b, d, f)$ are for $\xi_{s}=1 / 9$, which corresponds to global models of the $\mathrm{H}_{2}-\mathrm{O}_{2}$ reaction. For this stoichiometric ratio the flame is near the edge of the mixing layer, in the interface between mixed and unmixed fluid, and LES may be expected to have more problems. Plots $(a, b)$ are mass fraction profiles obtained from the relatively smooth function (1.5). Plots $(c, d)$ are profiles of $Y_{p}^{4}$, which is proportional to the fourth power of the temperature, and would therefore be a rough model for the source term of radiative heat in a flow with a real, hot, flame. This function is much sharper than $Y_{p}$ (figure $7 a$ ), and is therefore sensitive to the local values of the p.d.f., in spite of which the errors in the mean profile are still small. The last two plots $(e, f)$ are the values of the p.d.f. at a given $\xi_{s}$, and are the most sensitive test of the three. They are also the ones for which the errors are larger, but it is remarkable that the general form of the profile is still captured, and that the errors stay, at worst, of the order of $25 \%$.

For any profile which vanishes at $y= \pm \infty$ we can define a 'thickness'

$$
\delta_{f}=\int_{-\infty}^{\infty} f(y) \mathrm{d} y
$$

which is proportional to the total amount of the particular quantity contained in the layer, and which can be used to quantify the global error of the approximation. Note that this thickness is unchanged by the filtering, $\delta_{\bar{f}}=\delta_{f}$. The results for the different profiles of figure 6 are presented in figure $7(b)$, where they have been normalized with their DNS values. The errors for $h / \theta<1$ vary from better than $5 \%$ for the product mass fraction, to about $15 \%$ for the p.d.f. They are, as expected, generally larger for flames near the interface than for those at the centre of the mixed region.

\section{Finite rate effects}

If the speed of the chemical reaction is large but finite it is still possible to treat the combustion problem as a perturbation of the Burke-Schuman limit. In this 'flamelet' regime the deviations from infinitely fast chemistry are confined to a thin region 

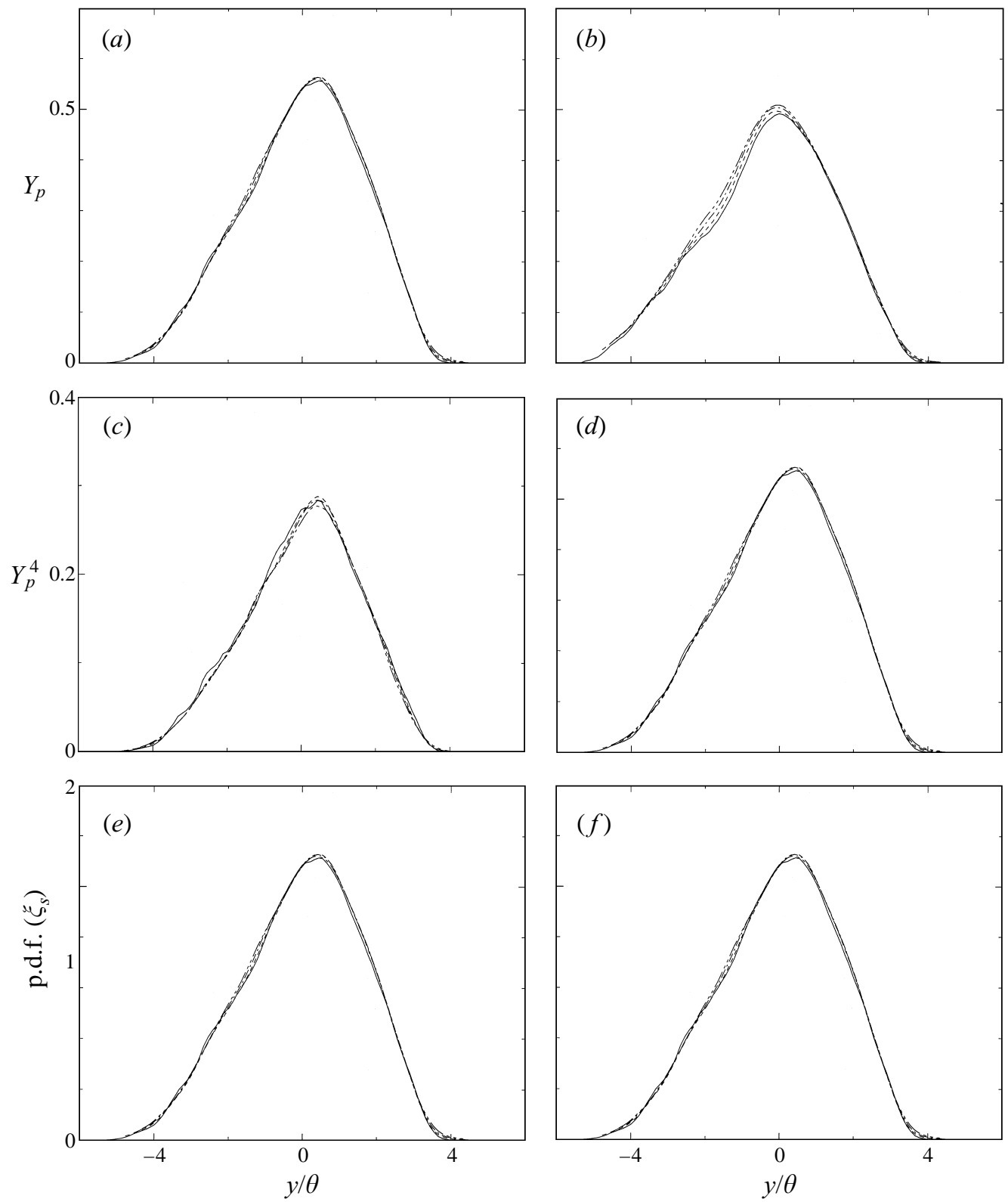

FIGURE 6. Average profiles for different functions in the forced layer. $(a, b)$ Product mass fraction $Y_{p} .(c, d)$ 'Radiation' source $Y_{p}^{4} .(e, f) \operatorname{PDF}\left(\xi_{s}\right) .(a, c, e)$ are for $\xi_{s}=1 / 2 ;(b, d, f)$ are for $\xi_{s}=1 / 9$. Lines as in figure 5 .

around the location of the stoichiometric mixture fraction, whose width is a function of the Damköhler number (Peters 1984).

Although the reaction zone is typically thin, there are cases in which the nonequilibrium effects are globally important. One such example is the $\mathrm{H}_{2}-\mathrm{O}_{2}$ reaction, in which an intermediate species is the $\mathrm{H}$ radical which, even in small amounts, controls the global exothermic properties. Another one is the thermal $\mathrm{NO}_{\mathrm{x}}$ production in air, which is controlled by the temperature and by the concentration of the $\mathrm{O}$ radical. $\mathrm{A}$ 

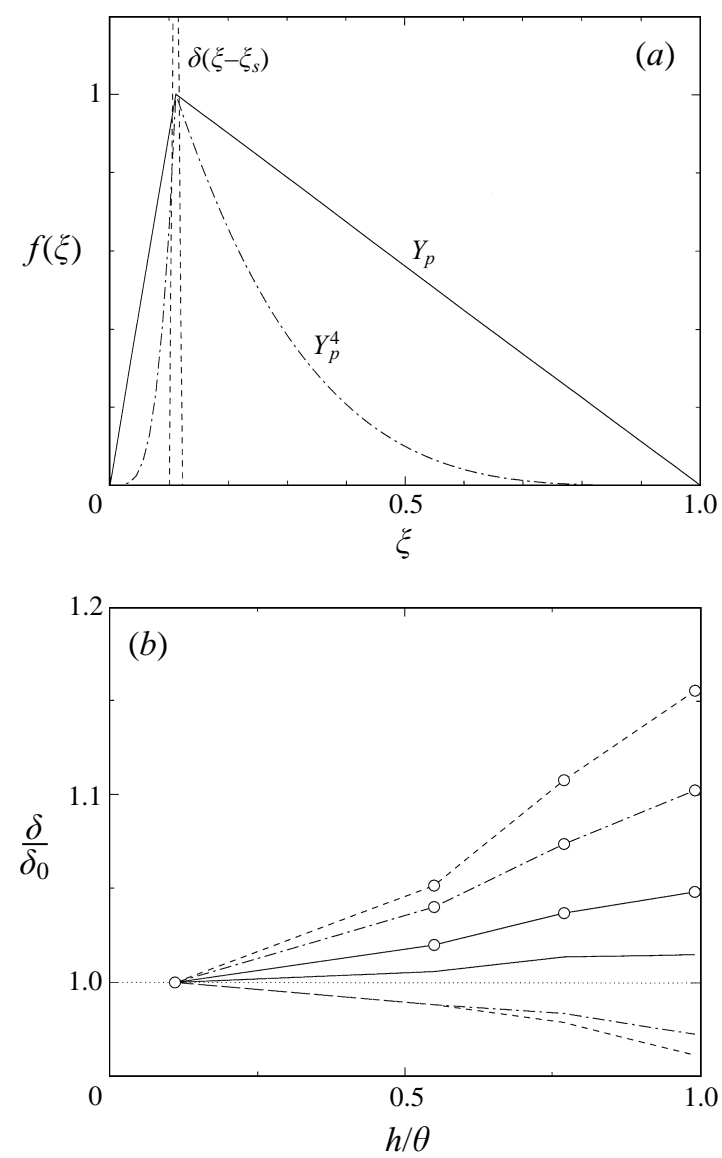

FIGURE 7. (a) The three functions used for the profiles in figure $6 . \xi_{s}=1 / 9$. (b) Relative variation of the profile thickness with filter width. Forced layer. Lines with symbols are for $\xi_{s}=1 / 9$. Other lines are for $\xi_{s}=1 / 2:-, Y_{p} ;-\cdot-, Y_{p}^{4} ;---$, delta function with $\Delta \xi=0.02$.

simplified scheme for the first example, involving two irreversible reactions

$$
3 \mathrm{H}_{2}+\mathrm{O}_{2} \rightarrow 2 \mathrm{H}_{2} \mathrm{O}+2 \mathrm{H}, \quad 2 \mathrm{H}+\mathrm{M} \rightarrow \mathrm{H}_{2}+\mathrm{M},
$$

was analysed by Sánchez et al. (1995). The first reaction is very fast, preventing $\mathrm{H}_{2}$ and $\mathrm{O}_{2}$ from coexisting at any given location, while the second one is slower, involving a characteristic recombination time $t_{r}$ which does not depend strongly on the temperature, and leading to finite reaction rate corrections. When the Damköhler number of this second reaction is large, the concentration of the radical is small and, in a first approximation, the two-step scheme can be simplified to an overall irreversible reaction $2 \mathrm{H}_{2}+\mathrm{O}_{2} \rightarrow 2 \mathrm{H}_{2} \mathrm{O}$, which is described in terms of a single mixture fraction $\xi$. In this case the first reaction occurs at a surface located very close to the stoichiometric one $\xi_{s}$. The radical is generated at that surface and remains confined to a thin recombination region surrounding it. The Damköhler number is defined as $D=\left(\kappa \psi_{s}^{2} t_{r}\right)^{-1}$, where $\psi=|\nabla \xi|$ is the gradient of the mixture fraction, which is computed here at the stoichiometric level. In this approximation the only flow-dependent variable is $\psi_{s}$, which therefore determines the structure of the flame.

It turns out that both the thickness and the maximum concentration in the radical 
containing region are proportional to $D^{-1 / 3}$, so that the total mass of $\mathrm{H}$ radical per unit flame area is proportional to

$$
m \sim D^{-2 / 3} \sim \kappa^{2 / 3} \psi_{s}^{4 / 3} .
$$

There is a chemical energy associated to this mass which leads to a lowering of the flame temperature.

The dependence on a power of the gradients is common to many other examples of slightly out-of-equilibrium reactions (Williams 1985), although the exponent changes from one case to another. Assume in general that $m \sim D^{-\alpha} \sim \kappa^{\alpha} \psi_{s}^{2 \alpha}$. We can estimate the average mass fraction of the radical by a procedure similar to that used in (1.6). If $S$ is the flame area per unit volume we write

$$
\left\langle Y_{H}\right\rangle=\int_{S} m \mathrm{~d} S
$$

which we wish to transform into a probability integral. Introduce the joint p.d.f. of $\psi$ and $\xi$, and define $\mathrm{d} n$ as the element of length normal to the flame, located at $\xi=\xi_{s}$. We can define the volume element either in terms of the geometry or of the p.d.f.,

$$
\mathrm{d} V=\mathrm{d} n \mathrm{~d} S=p_{2}(\psi, \xi) \mathrm{d} \psi \mathrm{d} \xi,
$$

from which, using that $\psi=\mathrm{d} \xi / \mathrm{d} n$, it follows that $\mathrm{d} S=\psi p_{2}(\psi, \xi) \mathrm{d} \psi$, and

$$
\left\langle Y_{H}\right\rangle=\int_{\xi=\xi_{s}} \psi m(\psi) p_{2}(\psi, \xi) \mathrm{d} \psi=p\left(\xi_{s}\right) \int_{0}^{\infty} \psi m(\psi) p\left(\psi \mid \xi_{s}\right) \mathrm{d} \psi .
$$

The new p.d.f. which appears in the second part of this equation, $p\left(\psi \mid \xi_{s}\right)$, is that of the gradient conditioned to $\xi=\xi_{s}$. Note that (4.5) has the same form as (1.6) with

$$
f(\xi)=C_{\psi} \delta\left(\xi-\xi_{s}\right), \quad C_{\psi}=\int_{0}^{\infty} \psi m(\psi) p\left(\psi \mid \xi_{s}\right) \mathrm{d} \psi,
$$

which is a delta function at the location of the flame, with a prefactor proportional to a moment of the conditional gradient p.d.f.

One of the referees pointed out that (4.5) can be used, in the particular case of $m=1$, to estimate the flame surface per unit volume,

$$
\Sigma=\mathrm{d} S / \mathrm{d} V=p\left(\xi_{s}\right) \int_{0}^{\infty} \psi p\left(\psi \mid \xi_{s}\right) \mathrm{d} \psi .
$$

This is a central quantity in many combustion models, and much work has been devoted to solving transport equations for it (Bray 1996), but it can simply be expressed as a quadrature within the present approximations.

Formulas of this type have been known for a long time in the context of nonequilibrium chemistry (Bilger 1976), and the joint p.d.f. of a scalar and its gradient has been the subject of intensive study. There is general consensus that the unconditional p.d.f. of the gradient is approximately log-normal (Kerstein \& Ashurst 1984; Anselmet \& Antonia 1985; Eswaran \& Pope 1988; Pumir 1994; Holzer \& Siggia 1994; Overholt \& Pope 1996), a form for which there is incomplete theoretical support (Gurvich \& Yaglom 1967; Meyers \& O’Brien 1981) but which seems to be only an approximation to the real one.

The largest deviations from log-normality occur in the tails of the distributions, which are better described by stretched exponentials which depend on the Reynolds number. This property is shared by the p.d.f.s of the turbulent energy dissipation, 
bringing into question the Reynolds number independence of the present results and of LES in general. There is an extensive literature on those small-scale intermittency effects, recently reviewed by Sreenivasan \& Antonia (1997), and the general conclusion is that low-order statistics, such as mean values and moments below approximately order 4, vary only weakly with Reynolds number. From the point of view of the present work, the highest-order quantity of interest is usually $C_{\psi}$ in (4.6), which is proportional to the moment of order $q=2 \alpha+1$ of the conditional p.d.f. The value of $q$ depends on the particular reaction, but $q=7 / 3$ for the case in (4.2).

There is less consensus on the conditional p.d.f. and, in particular, on whether the conditional variance of the gradient, $\psi_{\xi}^{\prime 2}=\int \psi^{2} p(\psi \mid \xi) \mathrm{d} \psi$, is correlated to the value of $\xi$. An early experiment, and one of the closest to our case, is that of Anselmet $\&$ Antonia (1985), who studied a heated jet and found the statistics of $\xi$ and $\partial \xi / \partial t$ to be essentially uncorrelated. Bilger (1989) reviewed the evidence up to that time and found examples both of dependence and independence. Pumir (1994) studied numerically the mixing by isotropic turbulence of a scalar with a mean gradient, and pointed out that the local scalar gradient is very intermittent, and that high values are concentrated in large-scale sheets, separated by 'ramps' in which the gradients are lower. This ramp and cliff structure had also been observed in mixing layers (Mungal \& Dimotakis 1984), where it is usually associated with the large coherent eddies. Jayesh \& Warhaft (1992) found it in grid turbulence acting on a mean scalar gradient, but pointed out that it is absent from partially premixed situations lacking a mean gradient. The cliffs seem to be associated with the advection of the global gradient by very large structures, and are sites both of large fluctuations of the scalar and of large local gradients. They are responsible in this way for a correlation between $\psi_{\xi}^{\prime}$ and $\xi$, which takes the form of a parabolic dependence of the former on the latter. Whenever the scalar fluctuations are small and their distribution is close to Gaussian, the gradients seems to be uncorrelated with them, but as soon as they develop large excursions and exponential tails, the parabolic correlation appears (Jayesh \& Warhaft 1992).

We have checked conditional gradient p.d.f.s for our two shear layers and the results are shown in figures 8 to 10 . It is seen in the first figure that the form of the p.d.f. is fairly independent of both $\xi_{s}$ and of the location in the flow, when each p.d.f. is referred to its own standard deviation. It has a general, but not exactly, log-normal shape, although our statistics are not good enough to give definite results on the behaviour of the tails. Note that the figure includes p.d.f.s conditioned on values of $\xi$ close to zero, but compiled at locations at which the mean value of $\xi$ is close to 1 . Those refer to gradients in the rare fluid particles which have managed to cross the whole layer without appreciable mixing, and they are a stringent test of the universality of the shape of the p.d.f.

Figure 9 presents a two-dimensional map of the conditional standard deviation, $\psi_{\xi}^{\prime}$, as a function of $\xi$ and of the location across the layer. It was found that the map was more uniform if $\psi_{\xi}^{\prime}$ was normalized with $\psi_{0}^{\prime}$, the unconditional standard deviation at the centre of the layer, than when the normalization was done with the unconditional $\psi^{\prime}$ at the particular $y$ location. The first choice is used in the figure. It is seen that $\psi_{\xi}^{\prime}$ has a central plateau in which it is approximately equal to $\psi_{0}^{\prime}$, but becomes larger near the edges of the layer, and vanishes at $\xi=0$ and $\xi=1$. The latter is an obvious property of laminar unmixed fluid and will be discussed below. The decline is, on the other hand, fairly local and only happens when $\xi$ is within $10 \%$ of the values for unmixed fluid. It should therefore not be important unless the stoichiometric $\xi_{s}$ is very close to 0 or 1 . The rise near the edges of the layer is real, but corresponds 


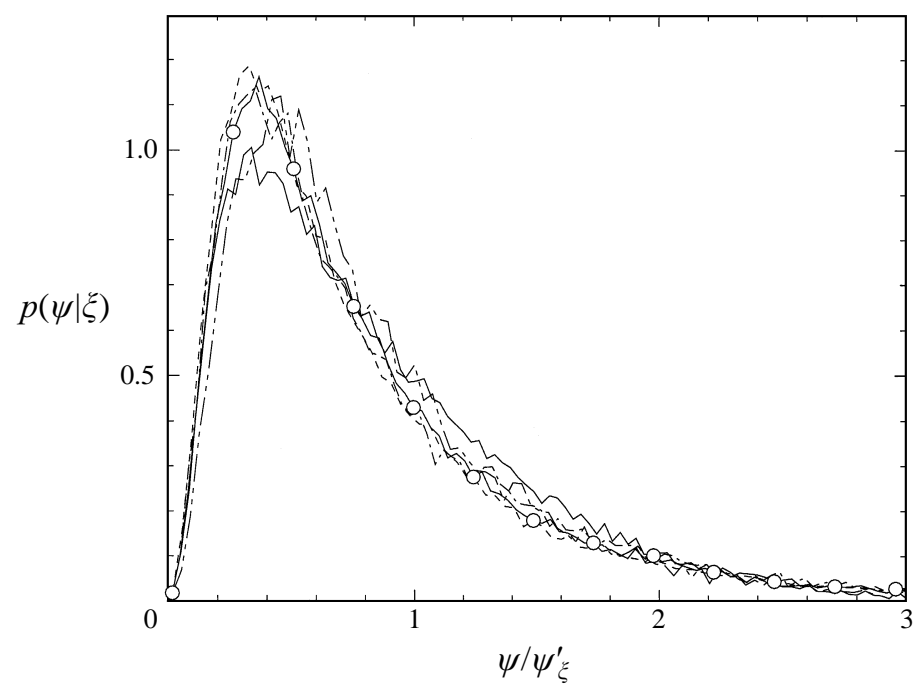

FIGURE 8. PDFs of the mixture fraction gradients conditioned on the mixture fraction, and compiled at different locations across the forced layer:,$- \xi=0.2 ;---, 0.5 ;-\cdot-, 0.8 ;-\cdot--, 0.2 ;-\circ-, 0.5$. The first three curves are for $y / \theta \in(-1,1),\langle\xi\rangle=0.46$. The last two are for $y / \theta \in(1,3),\langle\xi\rangle=0.72$.

to combinations of mixture fraction and location which are relatively improbable, as seen in figure $9(b)$, where the areas of high scalar gradients have been overlaid on a two-dimensional map of the mixture fraction p.d.f. It is clear that they correspond to events whose probability is mostly below $10 \%$ and which do not have a large weight in (4.5).

In figure 10 we have presented cross-stream profiles of $\psi_{\xi}^{\prime} / \psi_{0}^{\prime}$ for various values of $\xi$. These are essentially vertical cross-sections of figure $9(a)$, but they have been included to give quantitative information on the magnitude of the deviations of the scalar dissipation from its unconditional value, and to present data from the unforced layer. As in the previous figure it is seen that the scalar dissipation in the central part of the layer, where the fluid is well mixed, is roughly constant and equal to its unconditioned centreline value, but that the gradients conditioned on mixtures fractions close to the free-stream values, and all the gradients near the edges of the layer, have standard deviations that may differ from the global value by a factor of almost 2. They also have a characteristic parabolic shape. Most of these high deviations occur at places at which the absolute probability of $\xi$ is small, as seen in figures $10(b)$ and $10(d)$ which are conditioned on $p(\xi)>0.1$, and they will only have a small effect on (4.5), but the effect is real and of some theoretical interest. It brings immediately to mind the parabolic dependence of $\psi_{\xi}^{\prime}$ on $\xi$ that was mentioned above in the context of grid turbulence, and it probably has the same causes. We have mentioned already that the parabolic shape is associated with values of the mixture fraction near those of unmixed fluid, and located near the edge of the mixing layer. This is where one expects sharp fronts to appear between fluid from the free stream and the mixed turbulent region. In other words, the wings in figure 10 represent the initial mixing process of fresh fluid into the layer. Its relatively low weight in the global integral reflects the fact that this mixing is fast, happening in times of the order of the small-scale eddies, as compared with the turnover times of the large-scale structures.

In LES computations the value of $\psi_{0}^{\prime}$ cannot be estimated by similarity arguments 

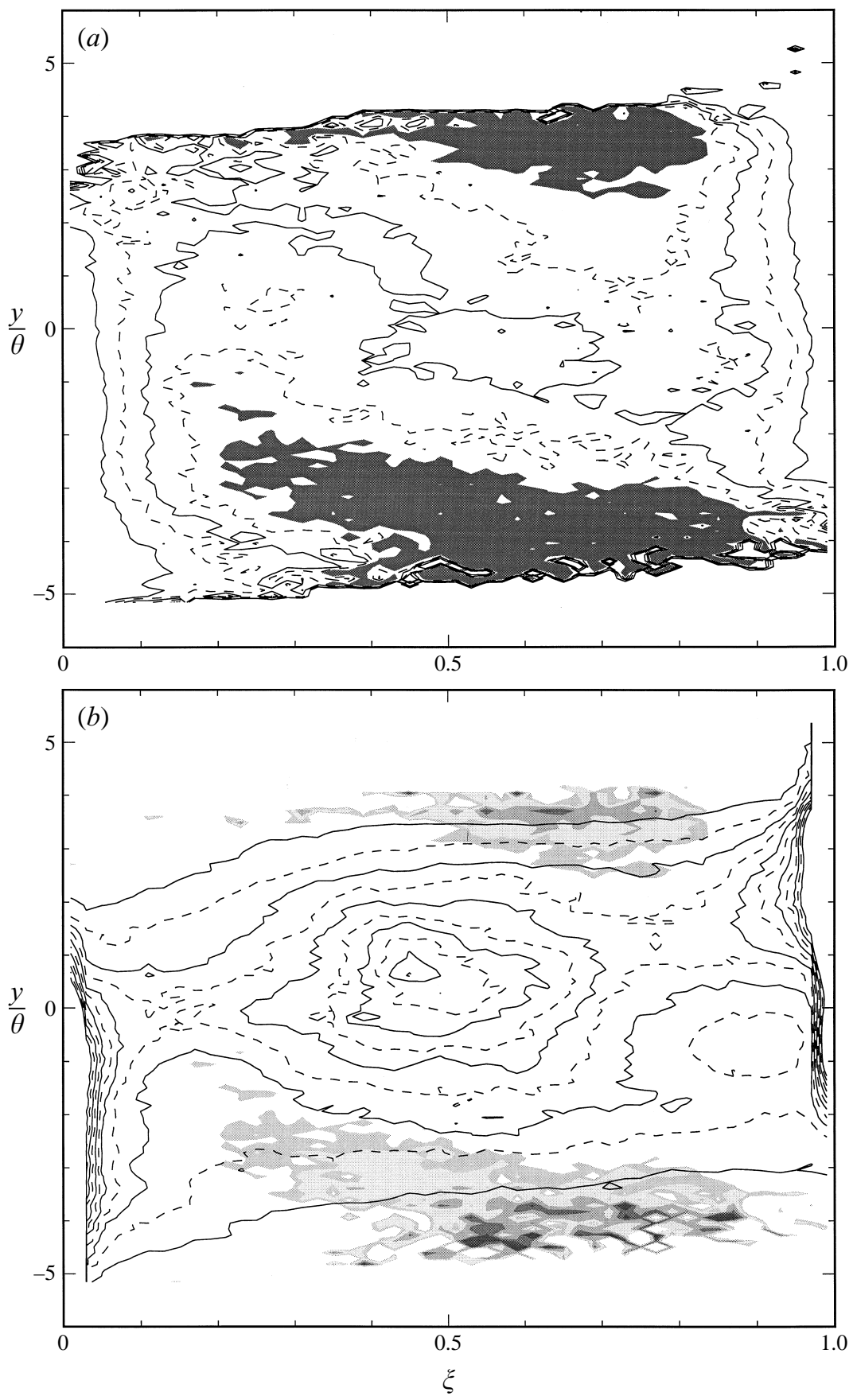

FIGURE 9. (a) Standard deviation of the gradient of the scalar as a function of $y / \theta$, conditioned on $\xi$ and normalized with the unconditional deviation at the central plane $\psi_{0}^{\prime}$. Isolines are $\psi_{\xi}^{\prime} / \psi_{0}^{\prime}=0.6(0.2) 1.2$, and shaded area is $\psi_{\xi}^{\prime} / \psi_{0}^{\prime} \geqslant 1$.4. Forced layer. (b) PDF of $\xi$ for the same flow. Each horizontal section represents the p.d.f. over one plane. Isolines $p(\xi)=0.1(0.2) 2.1$. Isolines alternate line style for clarity, and shaded regions correspond in both figures. 

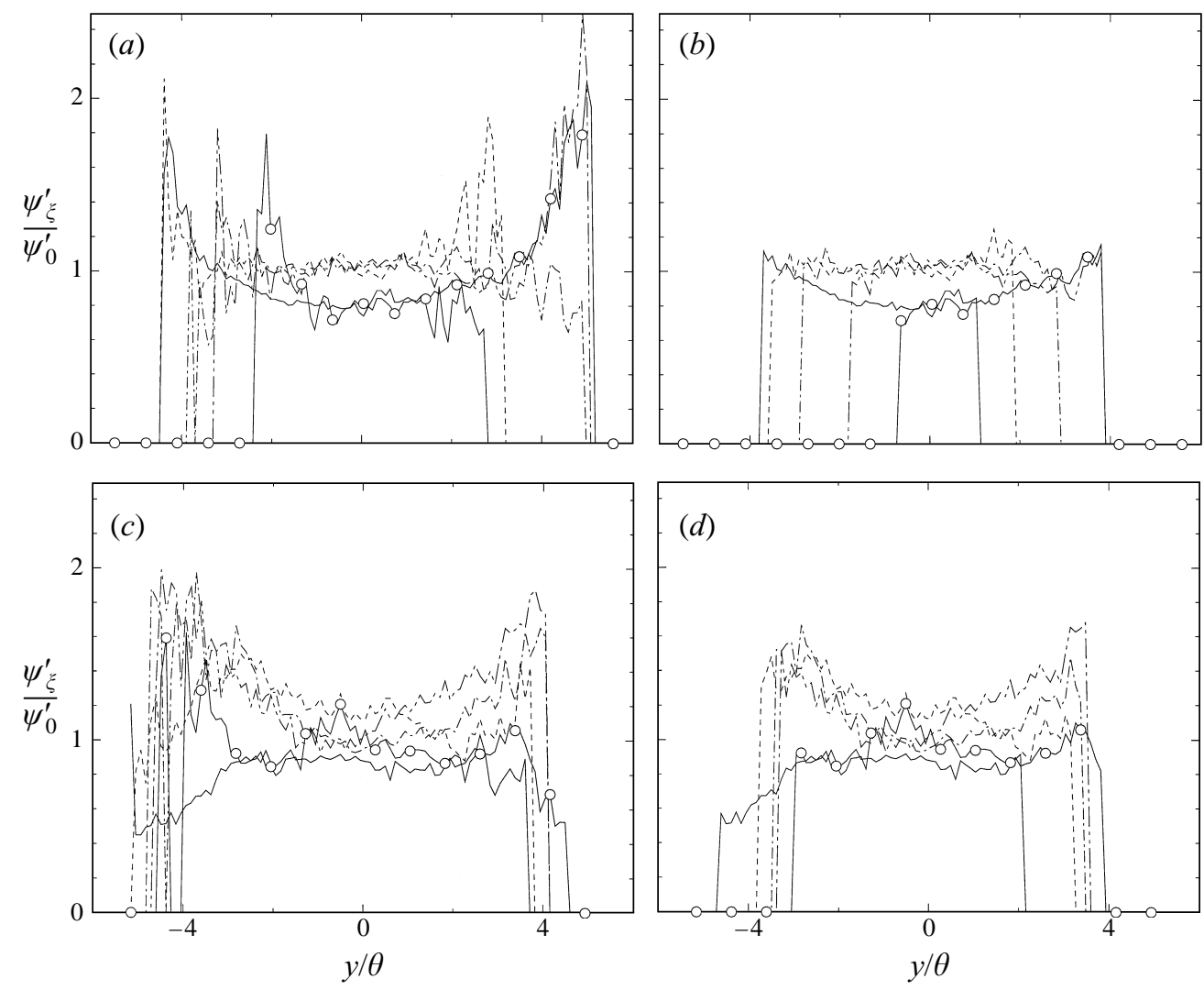

FIGURE 10. Variation across the layer of the standard deviation of the scalar gradient, conditioned on $\xi:-, \xi=0.1 ;---, 0.3 ;-\cdot-, 0.5 ;-\cdot-, 0.7 ;-\circ-, 0.9$. $(a, b)$ The unforced layer $;(c, d)$ the forced one; $(a)$ and $(c)$ present full profiles, but $(b)$ and $(d)$ are only for those points in which $p(\xi)>0.1$.

similar to those used for the subgrid scalar fluctuation. The problem is that while the spectrum of of $\xi$ decreases with wavenumber approximately like $S(k) \sim k^{-5 / 3}$, that for the gradient increases as $k^{2} S(k) \sim k^{1 / 3}$. Thus, while most of the contribution to $\xi^{\prime}$ comes from the resolved large scales, that to $\psi^{\prime}$ comes mostly from the unresolved small ones. In terms of the standard LES or modelling equations, $\xi^{\prime 2}$ is equivalent to the subgrid energy, while $\kappa \psi^{\prime 2}$ is equivalent to the subgrid dissipation. Conservation equations and closures for the subgrid scalar dissipation have been written by among others Newman, Launder \& Lumley (1981) and Elgobashi \& Launder (1983), and this quantity has often been simply assumed proportional to the turbulent energy dissipation rate (Bray \& Peters 1994). More recent closures have been proposed by Kerstein (1991) and Fox (1995).

It is interesting to note that the general magnitude of the gradients is low. The value of $\psi_{0}^{\prime} \theta$ is 1.5 for the unforced case and 2.0 for the forced one, so that the peak of the distributions in figure 8 is for gradients of the order of those of the mean $\xi$ profile. From the discussion in the previous paragraph it follows that this is a finite-Reynolds-number effect since, integrating the $k^{-5 / 3}$ spectrum, we obtain that $\psi^{\prime} \sim \operatorname{Re}_{\theta}^{1 / 2} / \theta$. High gradients are important because they lower the Damköhler number and may lead to local extinction of the flame, but we have seen above that they only appear in the form of the scalar dissipation, $\chi=\kappa \psi^{\prime 2}$, and it follows 


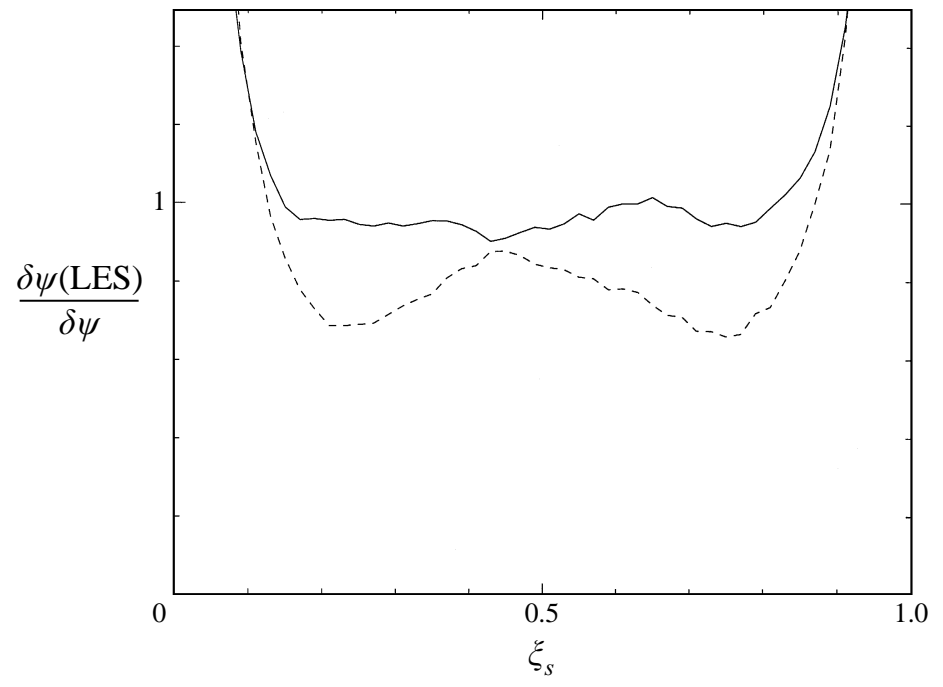

FIGURE 11. Computed scalar dissipation thickness, as defined in text, scaled by its DNS value. $h / \theta \approx 0.9$ : - , unforced layer; - - - , forced.

from the above estimates that this quantity is independent of the Reynolds number, $\chi \sim S c^{-1} \Delta U / \theta$. The results in figure 8 imply that the proportionality constant in this equation is of order unity, and that the diffusion times in the turbulent reaction are of the same order as the eddy-turnover time of the large-scale turbulent structures.

The 'engineering' consequences of the errors due to the gradient p.d.f.s are summarized in figure 11. Assume that we are interested in computing the total amount of $\mathrm{H}$ radical in the example (4.1), and that we use as a subgrid model everywhere a p.d.f. for the gradient whose shape is taken from figure 8 , scaled with a single unconditional $\psi_{0}^{\prime}$ estimated for the centre of the layer. Using (4.5) and integrating as in (3.8), we obtain a 'radical thickness' proportional to the integral across the layer of the $2 \alpha+1$ moment of the conditional p.d.f. of the gradients, weighted with the p.d.f. of the stoichiometric mixture fraction. This can be compared with the result of using the true gradients, and gives a global error due to the simplified assumptions on the p.d.f.s. The result depends on the exponent $\alpha$ but it is particularly simple in the case of $\alpha=1 / 2$, since the $2 \alpha+1$ moment is then proportional to the scalar dissipation, and the integral can be obtained directly from the data in figure 9 . In this case the approximation is equivalent to taking everywhere $\psi_{0}^{\prime}$ as an approximation to $\psi_{s}^{\prime}$, since it is clear from figure 8 that the assumption of a uniform shape is adequate. This normalized thickness is not very different from the results for $\alpha=2 / 3$, and is presented in figure 11. It is seen that, because the deviations from a universal distribution are mostly associated with places in which $p\left(\xi_{s}\right)$ is small, the final errors are reasonable in most cases, especially for the unforced case, but that they become $O(1)$ when the stoichiometric ratio approaches 0 or 1 .

The reason for this failure is clearly that we have not taken into account that the gradient has to vanish when the scalar is very near its maximum or minimum value. This is clear in figure $9(a)$, and it is interesting to estimate the range of $\xi_{s}$ for which a correction needs to be applied. It follows from the form of the scalar spectrum that, for $S c=O(1)$, most of $\psi^{\prime}$ is associated with scales of the order of the Kolmogorov length $\eta$, and that the scalar fluctuations at length $\ell$ are $\Delta \xi_{\ell} \approx \xi^{\prime}\left(\ell / L_{\epsilon}\right)^{1 / 3}$, where $L_{\epsilon}$ 
is an integral length. Since $L_{\epsilon} / \eta \approx R e_{\lambda}^{3 / 2}$ (Tennekes \& Lumley 1972), it follows that the scalar fluctuations which carry the gradients are of order

$$
\Delta \xi \approx \xi^{\prime} R e_{\lambda}^{-1 / 2}
$$

which in our case is 0.03 . As long as $\xi_{s}$ and $1-\xi_{s}$ remain large with respect to this value, the small eddies should not be affected by their proximity to the level of the unmixed fluid, but if they are of the same order as (4.8), large gradients become impossible. This suggest that the width of the lateral bands in figures $9(a)$ or 11 , which in our simulations is of the order of $\Delta \xi \approx 0.1$, should decrease as the Reynolds number increases. It would be interesting to get experimental confirmation of this estimate.

\section{Conclusions}

We have shown that relatively simple subgrid models for the p.d.f. of a conserved scalar can be used to obtain useful engineering approximations to global quantities in LES simulations of reacting non-premixed turbulent shear flows in the fast-chemistry limit. This is true even when the flow, in our case two different mixing layers, contains substantial intermittency.

The magnitude of the approximation error varies from less than $5 \%$ for the total amount of generated products, to about $15 \%$ for the p.d.f. of the scalar itself. When finite reaction rate corrections are introduced in the flamelet limit, the model has to be extended to include the p.d.f. of the scalar gradient, conditioned on the value of the scalar. We have shown that those p.d.f.s have an approximately universal form and that they can be expressed in terms of a single parameter, the conditional scalar dissipation, which varies little except at places in which the probability of finding mixed fluid is low. It is possible in those cases to obtain the global concentration of intermediate products (e.g. radicals) with errors which stay in the $20-30 \%$ range, except for reactions with stoichiometric mixture fractions very near those of the free streams. We have argued that the range of stoichiometric ratios for which the approximation fails should decrease with increasing Reynolds numbers. It should be clear however that, even within this range, the integrated quantities such as product concentration or radiation thickness are well predicted (figure 7).

The particular approximation used in our experiments is the $\beta$-model of Cook \& Riley (1994), but it is clear from the lack of correspondence between actual and assumed p.d.f.s, that other models might work as well. This also follows from similar observations of Cook \& Riley in their paper, and contrasts with the situation in RANS, where it is known that good subgrid models have to be used for the assumed p.d.f. if any but the simplest quantities are to be computed accurately (figure 5). It is important to understand the reason for this difference, which is essentially contained in figure 2, where the scalar p.d.f.s are reasonably well approximated even in the absence of a subgrid model. This means that most of the scalar fluctuations are associated with scales which are resolved by the LES, even for coarse grids like the ones used here. All that is left for the model is to correct situations in which the subgrid fluctuation is strong enough that the use of the average as a representation of the p.d.f. is no longer appropriate.

The situation would still be hopeless if those fluctuations were large enough to allow for a considerable latitude in the choice of subgrid p.d.f.s, but this is fortunately not the case. Consider the $\left(\bar{\xi}, \xi_{h}^{\prime}\right)$-plane in figure 12 . It can be shown that there can be no points above the dashed semicircle, and that p.d.f.s that fall on the semicircle 


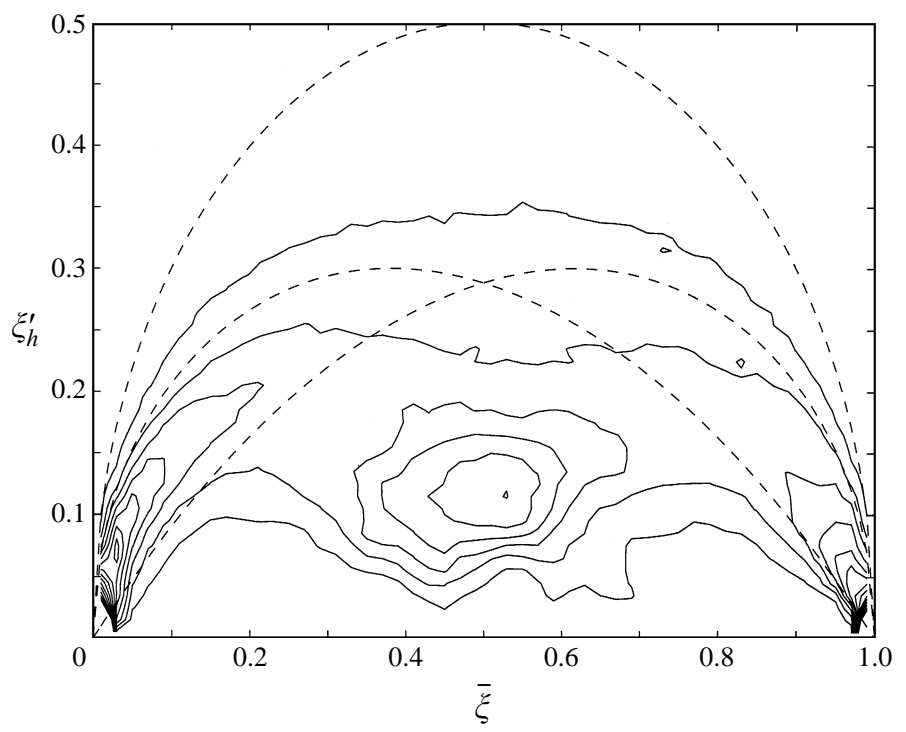

FIGURE 12. Joint p.d.f. for $\bar{\xi}$ and the subgrid fluctuation $\xi_{h}^{\prime}$. Forced layer. $h / \theta=0.88 . y / \theta \in(-2,2)$. Isolines are $p_{2}\left(\bar{\xi}, \xi_{h}^{\prime}\right)=1(2) 21$. Dashed semicircle is the limit of possible $\left(\bar{\xi}, \xi_{h}^{\prime}\right)$ combinations. Lower dashed lines are the limits below which the $\beta$-distribution looks like a single broadened spike.

must be formed exclusively by unmixed fluid with $\xi=0$ and $\xi=1$. In the same way, p.d.f.s on the horizontal axis are single delta functions of uniform fluid with $\xi=\bar{\xi}$. PDFs near that axis are roughly spread deltas, and those near the semicircle are spread bimodals. The border between the two cases varies for different models, but it is always near the two intermediate dashed lines in the figure, which correspond to the $\beta$-model. Below those lines, the p.d.f.s are bells, and almost all models should be equivalent. PDFs within the two crescents correspond to spread deltas near one or the other free stream, and are also easy to approximate, while p.d.f.s in the highfluctuation central part of the diagram are more difficult, and are likely to depend on more than two parameters.

We have overlaid on the diagram a typical joint p.d.f. for $\bar{\xi}$ and $\xi_{h}^{\prime}$, for a relatively wide filter in the intermittent 'hard' flow but, even in this case, most of the mass of the distribution is associated with p.d.f.s within the easily modelled parts of the diagram. The $\beta$-distributions form a flexible set of p.d.f.s which interpolate smoothly between the different cases, and they provide a simple numerical tool to evaluate the necessary integrals. This explains their practical success, but the reason why the approximation works lies in the small value of the subgrid standard deviations in figure 12. Since we have seen in figure 3 that these deviations can be estimated from large-scale quantities using the assumption of infinite Reynolds number for the upper limit of the spectrum, it is unlikely that much higher values might be found in other flows.

The small values of the fluctuations are also the reason why our relatively crude estimation of $\xi_{h}^{\prime}$ works so well. Even large errors in this estimation have relatively small effects on the final results, and some experiments in which the estimated subgrid fluctuations were systematically increased or decreased by $20 \%$ did not show appreciable differences with the results shown here.

This work was accomplished during the 1996 Summer School of the Centre for Turbulence Research. J.J., A.L. and F.J.H. were partially supported by the Instituto 
Nacional de Técnica Aeronáutica, under its combustion program. We thank P. Moin for many illuminating discussions and for bringing to our attention the work of Cook \& Riley.

\section{REFERENCES}

Anselmet, F. \& Antonia, R. A. 1985 Joint statistics between temperature and its dissipation in a turbulent jet. Phys. Fluids 28, 1048-1054.

BraY, K. N. C. 1996 The challenge of turbulent combustion. 26th. Symp. (Intl) on Combustion, pp. 1-26. The Combustion Institute.

Bray, K. N. C. \& Peters, N. 1994 Laminar flamelets in turbulent flames. In Turbulent Reactive Flows (ed. P. A. Libby \& F. A. Williams), pp. 64-113. Academic.

Bilger, R. W. 1976 The structure of diffusion flames. Combust. Sci. Technol. 13, 155-170.

Bilger, R. W. 1989 Turbulent diffusion flames. Ann. Rev. Fluid Mech. 21, 101-135.

BREIDENTHAL, R. E. 1981 Structure in chemically mixing layers and wakes using a chemical reaction. J. Fluid Mech. 109, 1-24.

Broadwell, J. E. \& Breidenthal, R. E. 1982 A simple model of mixing and chemical reaction in a turbulent shear flow. J. Fluid Mech. 125, 397-410.

Cook, A. W. \& Riley, J. J. 1994 A subgrid model for equilibrium chemistry in turbulent flows. Phys. Fluids 6, 2868-2870.

Dopazo, C. 1977 On conditional averages for intermittent turbulent flows. J. Fluid Mech. 81, 433-438.

Elgobashi, S. E. \& Launder, B. E. 1983 Turbulent time scales and the dissipation rate of temperature variance in the thermal mixing layer. Phys. Fluids 26, 2415-2419.

Eswaran, V. \& Pope, S. B. 1988 Direct numerical simulations of the turbulent mixing of a passive scalar. Phys. Fluids 31, 506-520.

Fox, R. O. 1995 The spectral relaxation model of the scalar dissipation rate in homogeneous turbulence. Phys. Fluids 7, 1082-1094.

Gurvich, A. S. \& Yaglom, A. M. 1967 Breakdown of eddies and probability distributions for small-scale turbulence, boundary layers and turbulence. Phys. Fluids Suppl. 10, S 59-65.

Holzer, M. \& Siggia, E. D. 1994 Turbulent mixing of a passive scalar. Phys. Fluids 6, 1820-1837.

JAYESH \& WARHAFT, Z. 1992 Probability distribution, conditional dissipation, and transport of passive temperature fluctuations in grid-generated turbulence. Phys. Fluids A 4, 2292-2307.

Kerstein, A. R. 1991 Linear-eddy modelling of turbulent transport. Part 6. Microstructure of diffusive scalar mixing fields. J. Fluid Mech. 231, 361-394.

Kerstein, A. R. \& Ashurst, W. T. 1984 Lognormality of gradients of diffusive scalars in homogeneous, two-dimensional mixing systems. Phys. Fluids 27, 2819-2827.

Kollman, W. 1984 Prediction of intermittency factors for turbulent shear flows. AIAA J. 22, 486-492.

Kollman, W. \& JanicKa, J. 1982 The probability density function of a passive scalar in turbulent shear flows. Phys. Fluids 25, 1755-1769.

KonRAD, J. H. 1976 An experimental investigation of mixing in two-dimensional turbulent shear flows with applications to diffusion-limited chemical reactions. PhD thesis, Caltech (CIT-8-PU).

Koochesfahani, M. M. \& Dimotakis, P. E. 1986 Mixing and chemical reactions in a turbulent liquid mixing layer. J. Fluid Mech. 170, 83-112.

LaRue, J. C. \& LibBy, P. A. 1974 Temperature fluctuations in the plane turbulent wake. Phys. Fluids 17, 1956-1967.

Lesieur, M. \& Rogallo, R. S. 1989 Large-eddy simulation of passive scalar diffusion in isotropic turbulence. Phys. Fluids A 1, 718-722.

Libby, P. A. 1975 On the prediction of intermittent turbulent flows. J. Fluid Mech. 68, 273-295.

Libby, P. A. \& Williams, F. A. (eds.) 1994 Turbulent Reacting Flows. Academic Press.

Lin, C. H. \& O'Brien, E. E. 1974 Turbulent shear flow mixing and rapid chemical reaction: an analogy. J. Fluid Mech. 64, 195-206.

Meyers, R. E. \& O’Brien, E. E. 1981 The joint p.d.f. of a scalar and its gradient at a point in a turbulent fluid. Combust. Sci. Technol. 26, 123-134. 
Moin, P., Squires, K., Cabot, W. \& LeE, S. 1991 A dynamic subgrid-scale model for compressible turbulence and scalar transport. Phys. Fluids 3, 2746-2757.

Mungal, M. G. \& Dimotakis, P. E. 1984 Mixing and combustion with low heat release in a turbulent shear layer. J. Fluid Mech. 148, 349-382.

Newman, G. R., Launder, B. E. \& Lumley, J. L. 1981 Modelling the behaviour of homogeneous scalar turbulence. J. Fluid Mech. 111, 217-232.

Overholt, M. R. \& Pope, S. B. 1996 Direct numerical simulation of a passive scalar with imposed mean gradient in isotropic turbulence. Phys. Fluids 8, 3128-3148.

Peters, N. 1984 Laminar diffusion flamelet models in non-premixed turbulent combustion. Prog. Energy Combust. Sci. 10, 319-339.

Pope, S. B. 1985 p.d.f. methods for turbulent reacting flows. Prog. Energy Combust. Sci. 11, 119-192.

PoPE, S. B. \& CORREA, S. M. 1988 Joint p.d.f. calculation of a non-equilibrium turbulent diffusion flame. 21st. Symp. (Intl) on Combustion, pp. 1341-1348. The Combustion Institute.

Pumir, A. 1994 A numerical study of the mixing of a passive scalar in three dimensions in the presence of a mean gradient. Phys. Fluids 6, 2118-2132.

RÉveillon, J. \& VervisCH, L. 1996 Response of the dynamic LES model to heat release induced effects. Phys. Fluids 8, 2248-2250.

Rogers, M. M. \& Moser, R. D. 1994 Direct simulation of a self-similar turbulent mixing layer. Phys. Fluids 6, 903-923.

Sánchez, A. L., Liñán, A., Williams, F. A. \& Balakrishnan, G. 1995 Theory of structures of hydrogen-air diffusion flames. Combust. Sci. Technol. 110, 277-301.

Sreenivasan, K. R. 1996 The passive scalar spectrum and the Obukhov-Corrsin constant. Phys. Fluids 6, 189-196.

Sreenivasan, K. R. \& Antonia, R. A. 1997 The phenomenology of small-scale turbulence. Ann. Rev. Fluid Mech. 29, 435-472.

Tennekes, H. \& Lumley, J. L. 1972 A First Course in Turbulence. MIT Press.

Williams, F. A. 1985 Combustion Theory, 2nd. edn. Benjamin-Cummings. 